University of Wollongong

Research Online

Faculty of Informatics - Papers (Archive)

Faculty of Engineering and Information

Sciences

$1-1-2006$

\title{
Modelling the induced force of attraction in electrorheological nanofluids
}

Barry J. Cox

University of Wollongong, barryc@uow.edu.au

Ngamta Thamwattana

University of Wollongong,ngamta@uow.edu.au

James M. Hill

University of Wollongong, jhill@uow.edu.au

Follow this and additional works at: https://ro.uow.edu.au/infopapers

Part of the Physical Sciences and Mathematics Commons

\section{Recommended Citation}

Cox, Barry J.; Thamwattana, Ngamta; and Hill, James M.: Modelling the induced force of attraction in electrorheological nanofluids 2006, 709-712.

https://ro.uow.edu.au/infopapers/2551

Research Online is the open access institutional repository for the University of Wollongong. For further information contact the UOW Library: research-pubs@uow.edu.au 


\title{
Modelling the induced force of attraction in electrorheological nanofluids
}

\author{
Abstract \\ The problem of electric-field induced force between spheres is fundamental to electrorheological fluids. \\ In this paper we summarize recent work, and in particular present an exact solution to the electrostatic \\ problem of dielectric spheres subject to an externally applied electric field. The numerical results are \\ compared to published experimental data and the solution is shown to accurately predict the low- \\ frequency experimental results at all measured interstices and particularly is applicable to nanosized \\ spheres in close proximity. This method is applicable to nonidentical spheres and results indicate a \\ genuine maximum in the attractive force between spheres exists, which depends on relative particle size \\ and the electrostatic permittivity of the particles and that of the medium. These maxima have not \\ previously been reported in the literature and the existing published work of the present authors \\ constitutes the first published material on this topic.

\section{Keywords} \\ nanofluids, induced, force, attraction, modelling, electrorheological \\ Disciplines \\ Physical Sciences and Mathematics

\section{Publication Details} \\ Cox, B. J., Thamwattana, N. \& Hill, J. M. (2006). Modelling the induced force of attraction in \\ electrorheological nanofluids. In C. Jagadish \& G. Lu (Eds.), International Conference On Nanoscience and \\ Nanotechnology (pp. 709-712). Brisbane, QLD: IEEE.
}




\title{
Modelling the Induced Force of Attraction in Electrorheological Nanofluids
}

\author{
Barry J. Cox*, Ngamta Thamwattana and James M. Hill \\ Nanomechanics Group, School of Mathematics and Applied Statistics, \\ University of Wollongong, Wollongong, NSW 2522, Australia \\ *Email: bjc949@uow.edu.au
}

\begin{abstract}
The problem of electric-field induced force between spheres is fundamental to electrorheological fluids. In this paper we summarize recent work, and in particular present an exact solution to the electrostatic problem of dielectric spheres subject to an externally applied electric field. The numerical results are compared to published experimental data and the solution is shown to accurately predict the low-frequency experimental results at all measured interstices and particularly is applicable to nanosized spheres in close proximity. This method is applicable to nonidentical spheres and results indicate a genuine maximum in the attractive force between spheres exists, which depends on relative particle size and the electrostatic permittivity of the particles and that of the medium. These maxima have not previously been reported in the literature and the existing published work of the present authors constitutes the first published material on this topic.
\end{abstract}

Keywords- Laplace's equation; electrostatic potential; spherical harmonics; electrostatic force; re-expansion method; electrorheological fluids

\section{INTRODUCTION}

It is well known that a dielectric body subject to an external electric field will become polarized with bound charge, and when two bodies are present they interact producing a perturbation to these bound charges. Also, when the bodies are appropriately aligned, an electrostatic force is generated between the two bodies. Wang, Peng, Lu and Wen [1] describe an experiment which measures the field-induced force between two spheres in close proximity. The procedure involved placing two $6.3 \mathrm{~mm}$ diameter spheres of $\mathrm{SrTiO}_{3}$ at interstices varying from 0.01 to $0.8 \mathrm{~mm}$ and subjecting the spheres to an electric field and measuring the resulting force. The experiment confirmed the quadratic relationship between field strength and force of attraction predicted by electrostatic theory. However, they also found that the measured force of attraction at small interstices was much greater than that predicted by any of the models employed by that paper. The models used by Wang, et al. [1] were a dipole model as described by Coulson [2], a dipole model with local field corrections as presented by Davis [3], and a finite-element approach as described by Davis [4]. Wang, et al. [1] conclude that "it should be emphasized that all three models are no longer valid for the case of a very thin gap" and "this result indicates that these available calculations for the mutual forces should be improved when the spheres are closely arranged." Cox, Thamwattana and Hill [5] employ the multipole re-expansion method around an axis of symmetry to calculate the force of attraction for the experimental configuration of Wang, et al. [1]. The authors' calculations showed that this approach accurately predicted the field induced force of attraction at all interstices and particularly addressed the short-comings of the other models at small interstices.

Electrorheological (ER) fluids, sometimes referred to as "tunable materials" because of their capacity to "tune" into any desired degree of solidity, comprise a mixture of small dielectric particles uniformly distributed throughout a fluid and through which an applied electric field operates. The electric field induces dipolar charges in the particles which results in an electrostatic force of attraction between them. When this force is sufficiently strong the "fluid" changes into a "solid", a process which is immediately reversible when the electric field is switched off. Such materials have traditionally had applications in areas such as clutches (Fig. 1) brakes, dampening devices, hydraulic valves (Fig. 2), shock absorbers and robotic control systems [6]. More recently proposed applications include gripping devices, human muscles simulators and spacecraft deployment dampeners [7]. Since

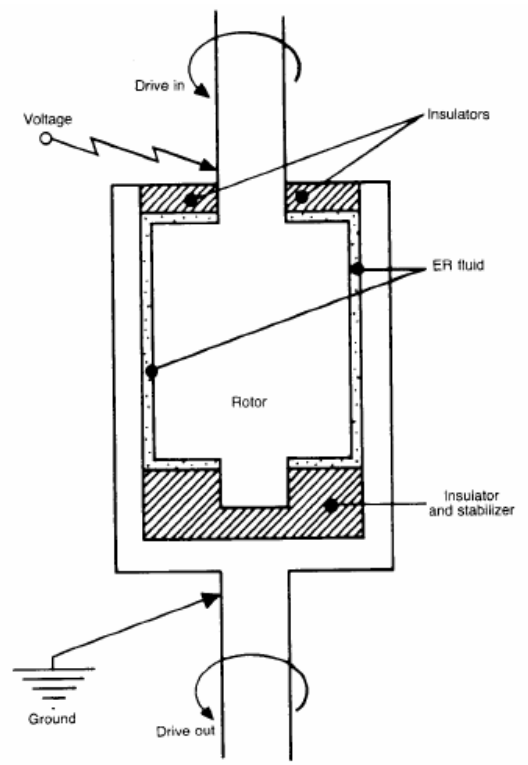

Figure 1. Example of a clutch based on ER fluids [6] 


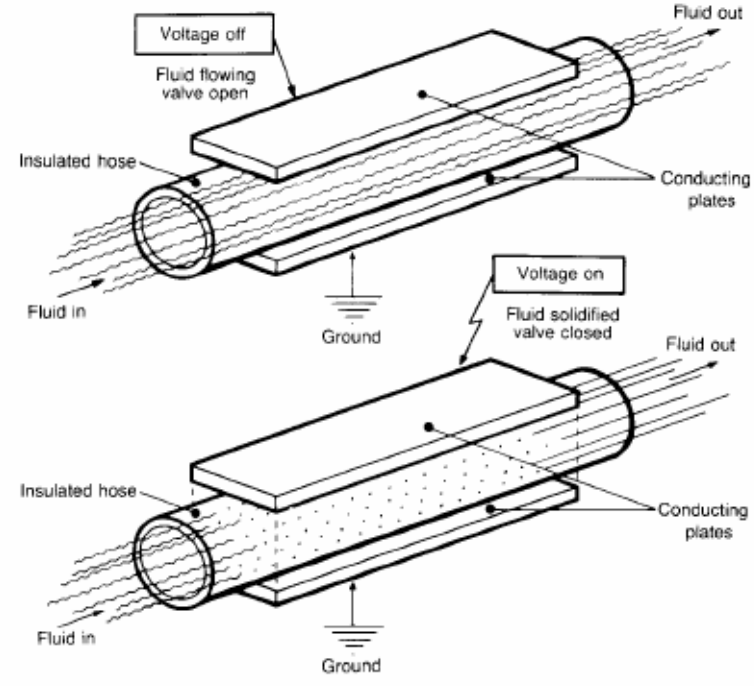

Figure 2. Example of a hydraulic valve based on ER fluids [6]

their discovery by Winslow [8] in the 1940s, many researchers have been working on various ways to maximize the ER effect. For example, Wen, et al. [9] report that by using nanoparticles one achieves the "giant ER effect." Another area of research has involved examining fluids in which the particles are of various sizes and mixed in various proportions to see how this affects the ER response. In the area of two different particle dimensions (bidisperse ER fluids) theoretical and experimental articles have been published but with seemingly conflicting results. The theoretical conclusions of Ota and Miyamoto [10] and experimental results of $\mathrm{Wu}$ and Conrad [11] suggest that bidispersity reduces the ER effect. However, See, Kawai and Ikazaki [12] show experimentally that bidispersity leads to an increased ER effect. Here we report that the mutual force of attraction between two nonidentical spherical dielectric particles in an electric field can be calculated from an analytical solution which is valid for various materials, gap distances and ratios of particle radii and which shows that for all combinations of variables, there exists a "magic" ratio of particle radii which produces a genuine maximum in the force of attraction between two nonidentical particles. This critical ratio varies substantially and depends on the interstice between, and the dielectric properties of, the particles. However, for a particular set of variables, the results can be used by ER designers to determine the critical ratio to employ in a bidisperse electrorheological fluid which will maximize the ER effect, and this by necessity must be the starting point for all designers of ER fluids. The full mathematical details are described by the authors' full article [13].

\section{METHOD}

The effectiveness of any ER fluid hinges on the magnitude of the electrostatic force of attraction between polarized dielectric particles, and therefore we are particularly interested in any circumstances which maximize this force. The multipole re-expansion method originally proposed by Washizu [14] has been used in [13] to solve the problem of two nonidentical dielectric spheres subject to an externally applied electric field. This method has been employed by a number of authors since it was first proposed but surprisingly a solution using this method for the problem of two uncharged, nonidentical spheres had not previously appeared in the literature. The method involves deriving solutions to Laplace's equation in spherical harmonics using distinct spherical coordinate systems located at the centre of each particle. Re-expansion formulae are then employed to re-expand the solution for the field perturbation caused by one particle, around the centre of the other particle. This then leads to a matrix solution which can be used to determine the coefficients of each term in the spherical harmonic expansions. An infinite series expression for the mutual electrostatic force of attraction experienced by each particle in terms of these coefficients can then be derived. For the full mathematical details refer to the authors' full articles $[5,13]$.

Once the matrix equation is determined a computer program written in $\mathrm{C}++$ and using the Template Numerical Toolkit (TNT) is employed to express and solve the matrix equation. At this point the matrix must be fixed in size to a system of $N$ equations, in $N$ unknowns. The value of $N$ employed is a function of the convergence for the particular variables employed. In general the higher the dielectric constant, the smaller the interstice and the larger the ratio of particle radii, then the larger the value of $N$ is required to produce a convergent result. The authors employed values of $N$ in the range from 50 to 800 , depending on the convergence requirements for the particular set of variables under consideration.

\section{RESULTS}

Figure 3 shows the results from the original experiment reported by Wang, et al. [1] as well as those calculated using the multipole re-expansion method. The vertical axis shows the force of attraction $f$ normalized by the square of the electric field strength $E_{0}$. The horizontal axis is a measure of sphere separation $\delta$. It can be seen that the re-expansion method

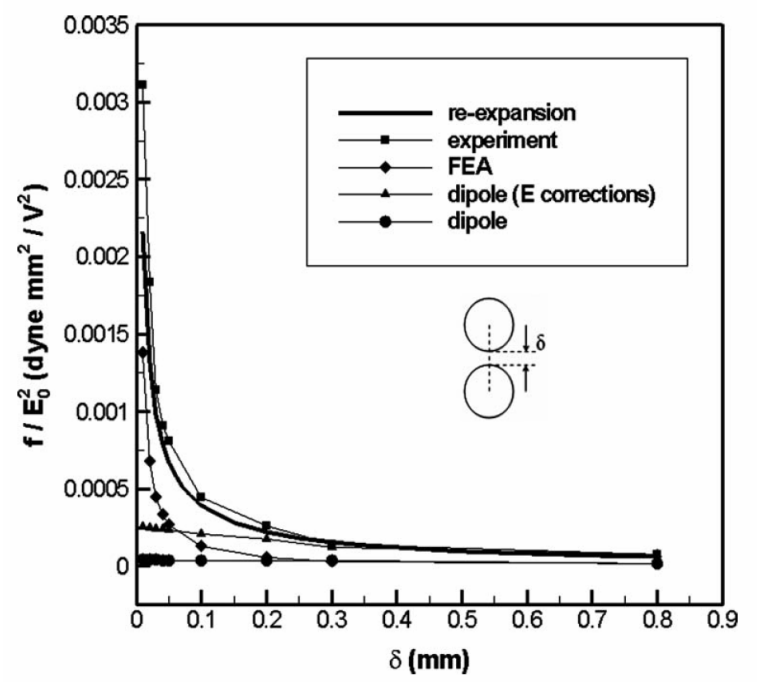

Figure 3. Comparison between the results originally reported by Wang, et al. [1] and the present exact solution. 


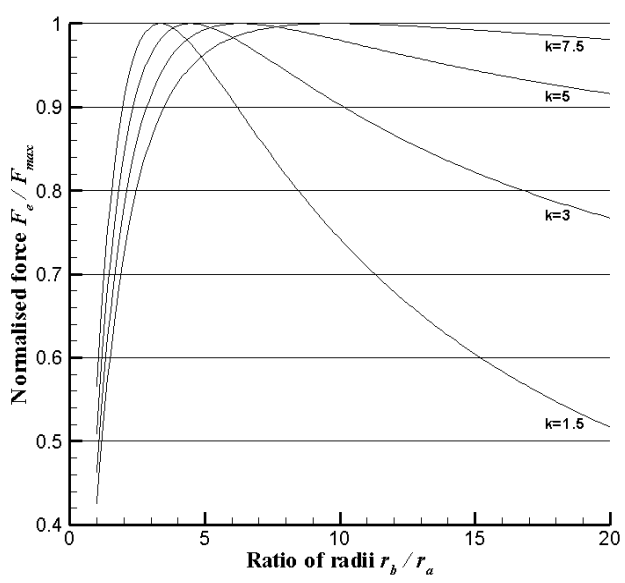

Figure 4. Comparison of mutual force for touching spheres varying the dielectric constant $k$ and the ratio of particle radii $r_{b} / r_{a}$. The force has been normalised using the maximum value reached for each value of $k$.

achieves a high level of agreement with the experimental results. Although at low interstices the agreement seems not quite so strong, it should be noted that the gradient of the graph in this region is such that any measurement error in the size of the interstice is likely to have a significant impact on the measurement of the mutual force of attraction. In any case, the predictions of the re-expansion method are a significant improvement on the three calculations which were used in the original comparison.

Figure 4 shows the electrostatic force on a particle when its size is held fixed while the radius of the other particle is varied from 1 to 20 times that of the first. Here the particles are assumed to be in contact and four different values of dielectric constant $k$ are shown. This illustrates the profile of force strength versus particle size ratio for low values of the

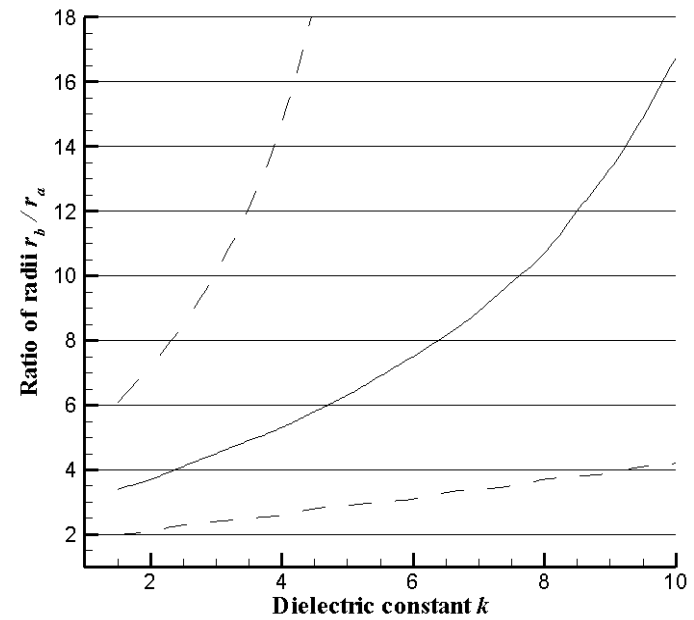

Figure 5. Plot of ratio of radii $r_{b} / r_{a}$ which leads to the maximum mutual force for two spheres in contact. Lower and upper bounds for $90 \%$ of the maximum force are also shown.

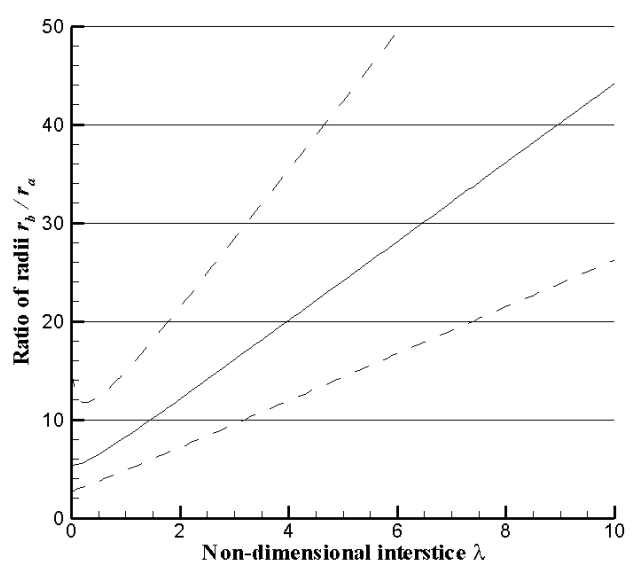

Figure 6. Plot of ratio of radii $r_{b} / r_{a}$ which leads to the maximum mutual force for two spheres at various interstices $\lambda$ and dielectric constant $k$ fixed to 4. Lower and upper bounds for $90 \%$ of the maximum force are also shown.

dielectric constant. For very low values there is a pronounced peak which also occurs at a lower ratio of radii $r_{b} / r_{a}$. As the dielectric constant increases the maximum force occurs at a higher ratio and the peak becomes less pronounced, especially for ratios higher than that of the maximum. We see clearly that if the larger sphere's radius is either increased or decreased from the optimal value, the resulting electrostatic force of attraction is lowered.

Figure 5 graphs the ratio of particle radii at which maximum force occurs for various dielectric constants $k$. Again the particles are assumed to be in contact and the dielectric constant allowed to vary from 1.5 to 10 . The figure also shows the lower and upper bounds of ratios where the force is $90 \%$ of the maximum possible value. As can be seen, for these moderate values of dielectric constant, a genuine optimal ratio

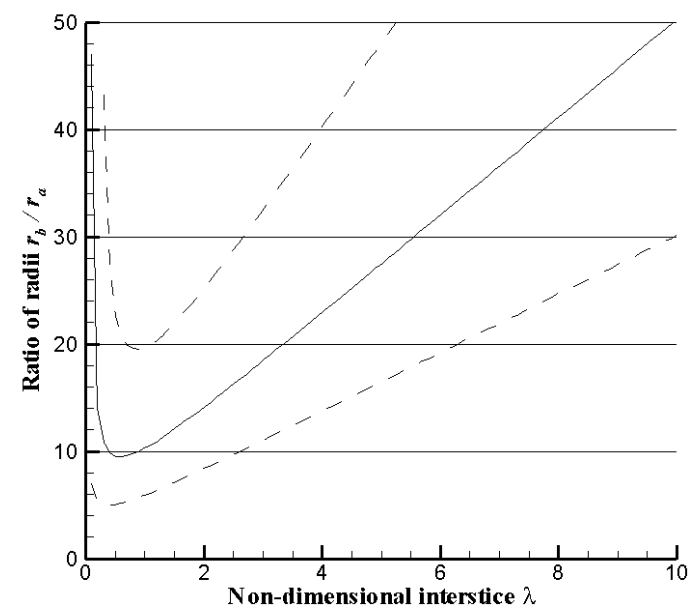

Figure 7. Plot of ratio of radii $r_{b} / r_{a}$ which leads to the maximum mutual force for two spheres at various interstices $\lambda$ and dielectric constant $k$ fixed to 300 . Lower and upper bounds for $90 \%$ of the maximum force are also shown. 
exists where the force between particles of different sizes is a maximum. This knowledge must be of considerable practical benefit in the design of electrorheological fluids.

Figure 6 graphs the ratio of radii at which the maximum force occurs for various non-dimensional interstices $\lambda=\delta / r_{a}$ with a dielectric constant $k$ of 4 . Here the interstice varies from 0 to 10 radii of the smaller sphere. The figure also shows the lower and upper bounds of ratios where the force is $90 \%$ of the maximum value. As can be seen, for this value of dielectric constant, as the distance between the spheres increases, so does the ratio of particle radii which leads to the maximum force of attraction between particles.

Figure 7 shows a graph of the ratio of radii at which maximum force occurs for various non-dimensional interstice $\lambda$ with a dielectric constant of 300 . Here the interstice varies from 0.1 to 10 radii of the smaller sphere. The figure also shows the lower and upper bounds of ratios where the force is $90 \%$ of the maximum value. In contrast to the previous graph, this graph shows that the point where the force is maximized for the smallest value of the ratio of particle radii does not occur at contact. There is a minimum distance of around 0.5 of a radius either side of which the optimum ratio of radii increases.

TABLE I. CRITICAL SPHERE RATIOS

\begin{tabular}{|c|c|c|c|c|c|c|c|}
\hline \multirow{2}{*}{$\boldsymbol{k}$} & \multicolumn{7}{|c|}{ Non-dimensional interstice $\boldsymbol{\lambda}$} \\
\cline { 2 - 8 } & $\boldsymbol{0}$ & $\boldsymbol{0 . 0 5}$ & $\boldsymbol{0 . 1}$ & $\boldsymbol{0 . 2}$ & $\boldsymbol{0 . 5}$ & $\boldsymbol{1}$ & $\boldsymbol{2}$ \\
\hline $\mathbf{1 . 5}$ & 3.35 & 3.52 & 3.69 & 4.02 & 5.02 & 6.68 & 10.02 \\
\hline $\mathbf{3}$ & 4.47 & 4.63 & 4.77 & 5.07 & 6.04 & 7.83 & 11.60 \\
\hline $\boldsymbol{6}$ & 7.48 & 6.99 & 6.73 & 6.58 & 7.10 & 8.77 & 12.70 \\
\hline $\mathbf{1 2}$ & $53.4^{*}$ & 13.03 & 10.17 & 8.48 & 8.03 & 9.43 & 13.37 \\
\hline $\mathbf{2 4}$ & & $73.9^{*}$ & 15.80 & 10.43 & 8.70 & 9.84 & 13.74 \\
\hline $\mathbf{5 0}$ & & & $45.5^{*}$ & 12.12 & 9.15 & 10.08 & 13.95 \\
\hline $\mathbf{1 0 0}$ & & & $47.6^{*}$ & 13.19 & 9.38 & 10.20 & 14.05 \\
\hline
\end{tabular}

Table I shows the ratio of radii $r_{b} / r_{a}$ which maximizes the force of attraction at various interstices $\lambda$ and various dielectric constant $k$. Note that for higher values of $k$ the table has been filled with approximate values (flagged with asterisks), due to the slower convergence mentioned earlier.

\section{CONCLUSIONS}

The solution used to derive these results is analytical in nature but like all solutions must be calculated using numerical techniques. It provides a solution to the problem described above and is similar to but different from that presented by Nakajima and Sato [15]. Firstly the results calculated using the multipole re-expansion method are shown to have a high level of agreement with experimental measurement and certainly provides an order of magnitude greater accuracy than other models for the situation where the particles are in close proximity. Secondly, by using this model and allowing the ratio of particle radii to vary, we predict that in an environment of modest dielectric constant $(1<k \leq 10)$, there exists an optimal ratio of particle radii which leads to a maximum value for the force between the particles at small interstices and in contact. Knowledge of the value at which this maximum occurs is crucial in the design of electrorheological nanofluids. Furthermore, this work suggests that well designed bidisperse nanofluids will exhibit a stronger electrorheological response than monodisperse fluids. For a full account of the mathematical details of this solution and more complete results readers are referred to the authors' papers $[5,13]$.

\section{ACKNOWLEDGMENT}

The support of the Australian Research Council through the Discovery Project Grant Scheme is gratefully acknowledged.

\section{REFERENCES}

[1] Z. Wang, Z. Peng, K. Lu and W. Wen, "Experimental investigation for field-induced interaction force of two spheres," Appl. Phys. Lett., vol. 82, pp. 1796-1798, 2003.

[2] C. A. Coulson, Electricity, $5^{\text {th }}$ ed., Edinburgh: Oliver \& Boyd, 1958.

[3] L. C. Davis, "Polarisation forces and conductivity effects in electrorheological fluids," J. Appl. Phys., vol. 72, pp. 1334-1340, 1992.

[4] L. C. Davis, "Finite-element analysis of particle-particle forces in electrorheological fluids,” Appl. Phys. Lett., vol. 60, pp. 319-321, 1992.

[5] B. J. Cox, N. Thamwattana, and J. M. Hill, "Electric field-induced force between two identical uncharged spheres," Appl. Phys. Lett., vol. 88, $152903,2006$.

[6] G. Goldstein, "Electrorheological fluids: applications begin to gel," Mech. Eng., vol. 112, pp. 48-52, 1990.

[7] T. Hao, "Electrorheological fluids," Adv. Mater., vol. 13, pp. 18471857, 2001.

[8] W. M. Winslow, "Induced fibration of suspensions," J. Appl. Phys., vol. 20, pp. 1137-1139, 1949.

[9] W. Wen, X. Huang, S. Yang, K. Lu and P. Sheng, "The giant electrorheological effect in suspensions of nanoparticles," Nature Mater., vol. 2, pp. 727-730, 2003.

[10] M. Ota, and T. Miyamoto, "Optimum particle size distribution of an electrorheological fluid,” J. Appl. Phys., vol. 76, pp. 5528-5532, 1994.

[11] C. W. Wu, and H. Conrad, "Influence of mixed particle size on electrorheological response," J. Appl. Phys., vol. 83, pp. 3880-3884, 1998.

[12] H. See, A. Kawai, and F. Ikazaki, "The effect of mixing particles of different size on the electrorheological response under steady shear flow," Rheol. Acta, vol. 41, pp. 55-60, 2002.

[13] B. J. Cox, N. Thamwattana, and J. M. Hill, "Maximising the electrorheological effect for bidisperse nanofluids from the electrostatic force between two particles," Rheol. Acta, vol. 45, pp. 909-917, 2006.

[14] M. Washizu, "Precise calculation of dielectrophoretic force in an arbitrary field," J. Electrostat., vol. 29, pp. 177-188, 1992.

[15] Y. Nakajima, and T. Sato, "Calculation of electrostatic force between two charged dielectric spheres by the re-expansion method," J. Electrostat., vol. 45, pp. 213-226, 1999. 